\title{
Generalised Mean Averaging Interpolation by Discrete Cubic Splines
}

\author{
By \\ Manjulata Shrivastava*
}

\begin{abstract}
The aim of this work is to introduce for a discrete function, certain discrete integrals which may reduce in particular to usual Riemann Stieltjes integrals. We name them as Discrete Stieltjes integrals. The existence and convergence of a discrete cubic interpolatory spline whose discrete Stieltjes integrals between consecutive meshpoints match with the corresponding integrals of a given periodic discrete function, are studied.
\end{abstract}

KEY WORDS: Discrete Stieltjes Integrals, forward differences, central differences, Discrete Splines.

\section{$\S 1$. Introduction}

Discrete integrals play a significant role in the theory of interpolation and approximation of functions defined on discrete subsets of the real line. Schumaker [8] and Lyche [4] have studied extensively the properties of discrete integrals. Here we introduce certain discrete integrals which we prefer to call Discrete Stieltjes (DS-) integrals, as they reduce in particular to the usual Riemann-Stieltjes integrals.

Schoenberg [7] and de Boor [1] have considered area matching interpolatory condition for even-degree splines. Considering Lebesgue integrals with respect to a non-negative measure, Sharma and Tzimbalario [9] have studied quadratic spline interpolants satisfying a fairly general mean-averaging condition. Similar interpolation problems for cubic splines and discrete cubic splines have been investigated in Dikshit [2] and Dikshit and Powar [3] respectively. Discrete splines are piecewise polynomials which satisfy smoothness requirements at knots in terms of differences. Our aim

Communicated by T. Kawai, March 31, 1992. Revised April 8, 1993.

1993 Mathematics Subject Classification: Primary 41 (A05), 65 (D07)

* Department of Mathematics and Computer Science, R.D. University, Jabalpur 482001India. 
in this paper is to study the existence and convergence properties of a discrete cubic spline whose discrete Stieltjes integrals between consecutive meshpoints match with the corresponding integrals of a given discrete function. For terms and notations we refer to [11].

\section{$\S 2$. Discrete Cubic Interpolatory Spline}

Given a real number $h>0$, let $f$ be a bounded function and $\alpha$ be a non decreasing function defined over a discrete interval $[a, b]_{h}$. The Discrete Stieltjes integral of $f$ with respect to $\alpha$ over $[a, b]_{h}$ is defined as:

$$
\int_{a}^{b} f(x) d_{h} \alpha(x)=\sum_{i=0}^{N-1} f(a+i h) \cdot[\alpha(a+(i+1) h)-\alpha(a+i h)],
$$

where it is assumed that $b-a=N h, N$ being a positive integer. The definition (2.1) remains valid if $\alpha$ is monotonic non-increasing, or in fact, if $\alpha$ is a function of bounded variation.

Let $P=\left\{x_{i}\right\}_{i=0}^{n}$ with $0=x_{0}<x_{1}<\cdots<x_{n}=1$, be a uniform sequence of points in $[0,1]_{h}$ such that $x_{i}-x_{i-1}=p, i=1,2, \cdots, n$. A discrete cubic spline with knots in $P$ is a piecewise cubic polynomial over $[0,1]$ which satisfies the conditions:

$$
\begin{aligned}
D_{h}^{\{j\}} s_{i}\left(x_{i}\right)=D_{h}^{\{j\}} s_{i+1}\left(x_{i}\right) \quad j & =0,1 \text { and } 2, \\
& i=1,2, \cdots n-1
\end{aligned}
$$

where $s_{i}$ is the restriction of $s$ in $\left[x_{i-1}, x_{i}\right]$ and $D_{h}^{\{j\}} g$ is the $j^{\text {th }}$ central difference of a function $g$. The space of discrete cubic splines with knots in $P$ is denoted by $S(4, P, h)$. Consider a non decreasing function $\alpha$ defined over $[0,1]_{h}$ such that

$$
\alpha(x+p)-\alpha(x)=K
$$

$K$ being a constant.

We shall investigate the following:

Problem 2.1. Given a 1-periodic discrete function $f$ over $[0,1]_{h}$, does there exist a unique 1-periodic discrete cubic spline $s$ in $S(4, P, h)$ satisfying the interpolatory condition 


$$
\int_{x_{i-1}}^{x_{i}}[f(x)-s(x)] d_{h} \alpha(x)=0, \quad i=1,2, \cdots, n ?
$$

A discrete cubic spline $s$ can be represented in terms of its second central differences at meshpoints, as follows:

$$
\begin{gathered}
6 p s(x)=M_{i-1}\left(x_{i}-x\right)^{\{3\}}+M_{i}\left(x-x_{i-1}\right)^{\{3\}}+6 c_{i}\left(x_{i}-x\right)+6 d_{i}\left(x-x_{i-1}\right) \\
x_{i-1} \leq x \leq x_{i}, \quad i=1,2, \cdots, n
\end{gathered}
$$

where $M_{i}=D_{h}^{\{2\}} s\left(x_{i}\right)$. Also, $c_{i}$ and $d_{i}$ are arbitrary constants, which in view of conditions $(2.2)$, are given by following relations

$$
\begin{aligned}
& d_{i}=c_{i+1} \\
& p^{2} M_{i}=d_{i-1}-2 d_{i}+d_{i+1}
\end{aligned}
$$

For convenience, we set

$$
\int_{x_{i-1}}^{x_{i}} f(x) d_{h} \alpha(x)=F_{i}, \quad \int_{x_{i-1}}^{x_{i}}\left(x_{i}-x\right)^{\{j\}} d_{h} \alpha(x)=A(j),
$$

and

$$
\int_{x_{i-1}}^{x_{i}}\left(x-x_{i-1}\right)^{\{j\}} d_{h} \alpha(x)=B(j) . \quad j=1,2, \cdots, n .
$$

In view of (2.3) we find that

$$
\begin{aligned}
& A(j)=\int_{x_{r-1}}^{x_{r}}\left(x_{r}-x\right)^{\{j\}} d_{h} \alpha(x) \\
& B(j)=\int_{x_{r-1}}^{x_{r}}\left(x-x_{r-1}\right)^{\{j\}} d_{h} \alpha(x) \quad r=1,2, \cdots, n ; \\
& j=1,2, \cdots
\end{aligned}
$$

and

$$
\int_{x_{i-1}}^{x_{i}} d_{h} \alpha=K=(1 / p)[A(1)+B(1)], \quad \text { for each } i .
$$

Thus, from interpolatory condition (2.4) we obtain the following 


$$
6 p F_{i}=M_{i-1} A(3)+M_{i} B(3)+6 d_{i-1} A(1)+6 d_{i} B(1) .
$$

Eliminating $d_{i}$ 's in (2.6) and the above equation we get

$$
\begin{aligned}
& B(3) M_{i+1}+\left[A(3)-2 B(3)+6 p^{2} B(1)\right] M_{i} \\
& +\left[-2 A(3)+B(3)+6 p^{2} A(1)\right] M_{i-1}+A(3) M_{i-2} \\
& =6 p\left(F_{i+1}-2 F_{i}+F_{i-1}\right), \quad i=1,2, \cdots, n,
\end{aligned}
$$

where $M_{n}=M_{0}, M_{n+1}=M_{1}, F_{n}=F_{0}$ and $F_{n+1}=F_{1}$. Now in view of the properties of Discrete Stieltjes integrals, it is easy to see that when $p>2 h$,

$$
\begin{aligned}
& A(3) \geq 0, B(3) \geq 0 \\
& p^{2} A(1) \geq A(3) \text { and } p^{2} B(1) \geq B(3)
\end{aligned}
$$

Therefore the coefficients of $M_{i+1}, M_{i}, M_{i-1}$ and $M_{i-2}$ are all nonnegative. Also, the excess of coefficient of $M_{i-1}$ over the sum of coefficients of $M_{i+1}, M_{i}$ and $M_{i-2}$ is

$$
\begin{aligned}
& 2\left[-2 A(3)+B(3)+3 p^{2}(A(1)-B(1))\right] \\
& =2 \int_{0}^{p}\left[p^{\{3\}}+3 x^{\{3\}}-6 p x^{2}+3 p h^{2}\right] d_{h} \alpha .
\end{aligned}
$$

Now if non-decreasing function $\alpha$ is such that it remains constant after $x=.466 p$ in each mesh interval then the expression $(2.8)$ is positive. The coefficient matrix of the system of equations (2.7) is then diagonally dominant and the system admits a unique solution.

Again, considering the excess of coefficient of $M_{i}$ over the sum of coefficients of $M_{i+1}, M_{i-1}, M_{i-2}$ we observe that if the function $\alpha$ is such that it remains constant upto $x=.533 p$ in each subinterval $[0, p]_{h}$, then the coefficient matrix of the system of equations (2.7) is invertible and the system is uniquely solved.

We have thus proved the following:

Theorem 2.1. Given a 1-periodic function $f$ and a non-decreasing function $\alpha$ defined over $[0,1]_{h}$ such that (2.3) holds, there exists a unique 1-periodic discrete cubic spline $s \in S(4, P, h)$ with $p>2 h$, satisfying (2.4) provided $\alpha$ is a function such that it remains constant either in $[.466 p, p]_{h}$ or in $[0, .533 p]_{h}$ for 
each subinterval $[0, p]_{h}$ of the mesh $P$.

\section{$\S 3$. Convergence}

Now we aim to establish the convergence properties of the discrete cubic spline interpolant of Theorem 2.1. Let $e=s-f$ denote the error function. We estimate the error-bounds in terms of 'discrete norm' and 'discrete modulus of smoothness' denoted by $\|f\|$ and $w(f, t)$ respectively (cf. [11]).

We shall prove the following:

Theorem 3.1. If $f, \alpha$, and $s \in S(4, P, h)$ be as in Theorem 2.1, then

and

$$
\left\|e_{i}^{\{2\}}\right\| \leq K_{1} w\left(f^{(2)}, p\right)
$$

where $K_{1}$ is a constant.

Proof of the theorem. Replacing $M_{i}$ in (2.7) by $e_{i}^{\{2\}}+f_{i}^{\{2\}}$, we have

$$
\begin{aligned}
& B(3) e_{i+1}^{\{2\}}+\left[A(3)-2 B(3)+6 p^{2} B(1)\right] e_{i}^{\{2\}} \\
& +\left[-2 A(3)+B(3)+6 p^{2} A(1)\right] e_{i-1}^{\{2\}}+A(3) e_{i-2}^{\{2\}}=6 p\left(F_{i+1}-2 F_{i}+F_{i-1}\right) \\
& -B(3) f_{i+1}^{\{2\}}-\left[A(3)-2 B(3)+6 p^{2} B(1)\right] f_{i}^{22\}} \\
& -\left[-2 A(3)+B(3)+6 p^{2} A(1)\right] f_{i-1}^{\{2\}}-A(3) f_{i-2}^{\{2\}} \equiv R(\text { say }) .
\end{aligned}
$$

Expanding $f(x)$ in each subinterval by Discrete Taylor formula we get

$$
F_{i}=f_{i-1} K+f_{i-1}^{(1)} B(1)+\theta_{i} f^{(2)}\left(z_{i}\right) \bar{B}(2)
$$

where $0 \leq \theta_{i} \leq 1, z_{i} \in\left(x_{i-1}, x_{i}\right)_{h} ;\left(x-x_{i-1}\right)^{(2)}=\left(x-x_{i-1}\right)\left(x-x_{i-1}-h\right)$ and

$$
\bar{B}(2)=\int_{x_{i-1}}^{x_{i}}\left(x-x_{i-1}\right)^{(2)} d_{h} \alpha(x) .
$$

We observe that

$$
f_{i-2}-2 f_{i-1}+f_{i}=p^{2} f^{(2)}\left(y_{i}\right)
$$

where $y_{i} \in\left(x_{i-2}, x_{i}\right)_{h}$; and

$$
\left|f_{i-2}^{(1)}-2 f_{i-1}^{(1)}+f_{i}^{(1)}\right| \leq 2 p w\left(f^{(2)}, p\right) .
$$


Therefore,

$$
|R| \leq 2\left[A(3)+B(3)+6 p \bar{B}(2)+6 p^{2} B(1)+3 p^{3} K\right] w\left(f^{(2)}, p\right) .
$$

If $\left|e_{j}^{\{2\}}\right| \geq\left|e_{i}^{\{2\}}\right|, i=1,2, \cdots, n$; then from (2.7) we have

$$
2\left[A(3)-2 B(3)+3 p^{2}(B(1)-A(1))\right]\left|e_{j}^{\{2\}}\right| \leq|R| .
$$

This directly leads to (3.1). It is easy to see from (2.5) that in $\left[x_{i-1}, x_{i}\right]$,

$$
p s^{\{2\}}(x)=M_{i-1}\left(x_{i}-x\right)+M_{i}\left(x-x_{i-1}\right) .
$$

Therefore,

$$
\begin{aligned}
p e^{\{2\}}(x)= & {\left[e_{i-1}^{\{2\}}+f^{\{2\}}(x)-f_{i-1}^{\{2\}}\right]\left(x-x_{i-1}\right) } \\
& +\left[e_{i}^{\{2\}}+f^{\{2\}}(x)-f_{i}^{\{2\}}\right]\left(x_{i}-x\right)
\end{aligned}
$$

A little calculation then leads to (3.2). This completes the proof of Theorem 3.1 .

Remarks.

1. In the case when $\alpha(x)=x$ and $h \rightarrow 0$, the mean averaging condition (2.4) reduces to the area matching condition considered in [10].

2. When $\alpha$ is a step function, for suitable choices of function $\alpha$, the interpolatory condition (2.4) reduces to different conditions of interpolation at one or more interior points in each mesh interval (cf. Meir and Sharma [6]). When $\alpha$ has a single jump at one end point in each mesh interval then the discrete cubic spline of Theorem 2.1 reduces to that considered in Lyche [5]. For an other appropriate choice of function $\alpha$ the interpolatory condition (2.4) reduces to the average-interpolation condition considered in [11].

3. The estimates (3.1) and (3.2) in Theorem 3.1 are sharp, i.e., as a functions of $n$, they decrease to zero, when $n \rightarrow \infty$ like $\beta \cdot n^{-1}$ where $\beta$ is a constant.

\section{References}

[1] de Boor, C., Appendix to splines and histograms; in Spline Functions and Approximation Theory, ISNM, Birkhauser Verlag, 21 (1973), 329-358.

[ 2 ] Dikshit, H.P., On cubic interpolatory splines; J. Approx. Theory, 22 (1978), 105-110. 
[ 3 ] Dikshit, H.P., Area matching interpolation by discrete cubic splines; Approx. Theory App. Res. Notes Math., 133 (1985), 35-45.

[ 4 ] Lyche, T., Discrete polynomial spline approximation methods, Report RRI2, University of Oslo (1975).

[ 5 ] - Discrete cubic spline interpolation, Report RRI5, University of Oslo (1975).

[6] Meir, A. and Sharma, A., Convergence of a class of interpolatory splines, J. Approx. Theory, 1 (1968), 243-250.

[ 7 ] Schoenberg, I.J., Splines and histograms, Spline Functions and Approximation Theory, ISNM, Birkhauser Verlag, 21 (1973), 277-327.

[ 8 ] Schumaker, L.L., Spline Functions: Basic Theory, John Wiley and Sons, New York 1981.

[ 9 ] Sharma, A. and Tzimbalario, J., Quadratic splines, J. Approx. Theory, 19 (1977), 183-193.

[10] Shrivastava, M., Cubic interpolatory splines matching the areas; Proc. Japan Acad., 67 (1991), 235-237.

[11] - Discrete cubic interpolatory splines, Publ. RIMS, Kyoto Univ., 28 (1992), 825-832. 
